\title{
Tendências de pesquisa em Aprendizagem Organizacional
}

\section{Research trends in Organizational Learning}

\author{
RENATO NEDER* \\ DIÓGENES DE SOUZA BIDO**
}

\section{RESUMO}

Este artigo tem por objetivo analisar os conteúdos semânticos dos artigos de Aprendizagem Organizacional com o intuito de mapear esse campo do conhecimento em administração. Para tanto, foi utilizada uma metodologia exploratória e longitudinal que teve como base 2.896 artigos da base de dados Web of Science. Os títulos e resumos foram analisados por meio da Análise de Redes Semânticas. Os resultados apontam possíveis contribuições da pesquisa em duas principais vertentes. A primeira é teórica, uma vez que a apreciação dos dados assinala agendas de pesquisa que potencialmente podem se destacar nas áreas de Aprendizagem Organizacional. Além disso, as teorias desenvolvidas auxiliam na explicação do desenvolvimento da área ao longo do tempo, além de tentar estimar seu futuro. A segunda contribuição é metodológica, uma vez que não foram encontrados artigos que utilizavam a metodologia proposta em textos de Aprendizagem Organizacional. Palavras-chave: Frentes de pesquisa; Aprendizagem Organizacional; Análise de Redes Semânticas.

* Universidade Federal de Mato Grosso. Doutor em Administração de Empresas pela MACKENZIE, Mestrado em Educação pela Universidade Federal de Mato Grosso, Graduação em Administração pela UFMT e professor do departamento de Administração da UFMT. renatoneder@hotmail.com .

** Universidade Presbiteriana Mackenzie, CCSA - Centro de Ciências Sociais e Aplicadas. Doutor em Administração de Empresas pela Universidade de São Paulo - FEA/USP, Mestrado em Administração de Empresas pela Universidade de São Paulo - FEA/USP, Graduação em Engenharia Química pela Escola Superior de Química Oswaldo Cruz das Faculdades Oswaldo Cruz, FOC, Brasil e professor do programa de doutorado em administração de empresas da Universidade Presbiteriana Mackenzie. diogenesbido@yahoo.com.br . 


\section{Abstract}

This article aims to analyze the semantic contents of the Organizational Learning articles in order to map this field of knowledge in administration. For this, an exploratory and longitudinal methodology was used, based on 2,896 articles from the Web of Science database. The titles and abstracts were analyzed through the Semantic Network Analysis. The results indicate possible contributions of the research in two main aspects. The first is theoretical, since the appreciation of the data points to research agendas that can potentially stand out in the areas of Organizational Learning. In addition, the theories developed help explain the development of the area over time, and try to estimate its future. The second contribution is methodological since no articles were found that used the methodology proposed in Organizational Learning texts.

Keywords: Research fronts; Organizational Learning; Semantic Network Analysis.

\section{INTRODUÇÃo}

A principal contribuição do artigo foi o aprofundamento teórico na área de Aprendizagem Organizacional (AO), em termos de suas agendas de pesquisa e potencialidades. Também teve a intenção de contribuir metodologicamente com o desenvolvimento de estudos nesse campo de conhecimento.

Para tanto, foi desenvolvida uma pesquisa exploratória quantitativa e bibliométrica, utilizando a metodologia de Análise de Redes Semânticas (ARSe). Foi analisado um conjunto de 2.896 artigos disponíveis na base de dados Web of Science, o que permitiu uma visão ampliada da produção cientifica da área ao longo do tempo, uma vez que o artigo mais antigo encontrado data de 1965, e o mais recente, de 2015.

Parte da importância deste artigo é que oportuniza a superação de algumas dificuldades e problemas inerentes às pesquisas na área, dentre as quais cabe destacar: a quantidade crescente de textos científicos; a dificuldade de analisar essa diversificada produção por meio das metodologias comumente utilizadas na área; e a dificuldade de se compreenderem as complexas relações presentes nesse aglomerado de textos científicos. 
A metodologia proposta permitiu identificar tendências da Aprendizagem Organizacional mediante as tendências de crescimento de suas subáreas e mapear prováveis frentes emergentes de pesquisa, que são subáreas que surgiram há pouco tempo nas redes de textos das áreas, mas apresentam uma importância crescente.

São várias as maneiras de conceituar o campo de AO. Parte-se então da concepção de Easterby-Smith e Lyles (2011), que se referem a ela como o estudo dos processos de aprendizagem no âmbito organizacional e que, na maioria das vezes, são pensados sob um ponto de vista acadêmico. Takahashi (2015) propõe que a Aprendizagem Organizacional ocorre quando estímulos externos levam a organização a reconfigurar seus comportamentos.

Propõe-se compreendê-la como processos de aprendizagem que possuem como uma de suas principais variáveis o espaço no qual a ação de aprendizagem ocorre - e esse espaço é o ambiente de trabalho. Dessa forma, compreender como indivíduos e grupos configuram e reconfiguram suas ações de aprendizagem pode ser importante para que as organizações dinamizem esses processos.

Por ser uma área do conhecimento que tem como foco principal a aprendizagem, múltiplas são as teorias, autores e modos de pensar. Essa diversidade, aliada à defasagem temporal dos textos e das metodologias utilizadas que não levam em conta o contexto de produção científica em larga escala, dificulta o desenvolvimento de teorias que possibilitem explicar a área de Aprendizagem Organizacional.

Um dos principais desafios encontrados é que o volume de informações representado pelas produções científicas da referida área é extenso e suas relações complexas, o que dificulta e, por vezes, até impossibilita um estudo aprofundado da $\mathrm{AO}$ e de suas relações por meio da análise de pequenos grupos de artigos (revisão sistemática da literatura). Para se ter uma ideia do problema, quando pesquisado o termo organizational learning no portal de periódicos da CAPES em 15/02/2016, foram encontrados 7.432 documentos. A mesma pesquisa no Google acadêmico resultou em 444.000 itens.

$\mathrm{Na}$ atualidade, o desenvolvimento e a circulação de informações crescem exponencialmente, por isso compreender como se dá a organização da produção científica de determinada área do co- 
nhecimento se mostra uma tarefa cada vez mais intrincada, porém relevante e necessária.

Nesse sentido, Groh e Fuchs (2011) apontam que a vasta quantidade de literatura existente, os diversos canais disponíveis e as inúmeras maneiras de publicar os trabalhos como livros, revistas, conferências, workshops, simpósios e páginas da web, entre outras, fazem com que seja cada vez mais difícil aos pesquisadores ficarem em contato com os resultados das investigações em curso.

Fujita et al. (2014) acrescentam que os métodos tradicionais, em que os pesquisadores fazem análises individuais dos textos, não conseguem lidar com os números crescentes de documentos e pesquisadores ou, ainda, são muito dispendiosos em termos de tempo e custos empregados. Dessa forma, é naturalmente difícil para os pesquisadores detectarem e compreenderem as relações presentes nesses aglomerados de estudos, artigos e outros textos.

Para Shibata e Kajikawa (2011), a quantidade de conhecimento acadêmico está aumentando tão rapidamente que as abordagens tradicionais não têm a capacidade de capturar a estrutura inteira de um domínio de conhecimento específico. Como alternativa, indicam recentes esforços que usam metodologias baseadas em computação para "capturar" e analisar grandes quantidades de conhecimento. Para eles, essas metodologias não são apenas escaláveis, mas também podem proporcionar estruturas comuns de pesquisa em várias frentes.

As múltiplas abordagens e perspectivas, aliadas ao contexto de produção científica em larga escala da contemporaneidade, dificultam o desenvolvimento de teorias que sejam amplamente aceitas entre os pesquisadores da área de Aprendizagem Organizacional, fator que justifica, em parte, as pretensões deste artigo.

Propõe-se como objetivos do artigo: mapear a área de Aprendizagem Organizacional ao longo do tempo por meio do estudo dos artigos correspondentes; buscar tendências (de conteúdo) nas produções científicas em AO; e identificar possíveis tópicos emergentes de pesquisa na área.

\section{REFERENCIAL TEÓRICO}

O conhecimento e por consequência a aprendizagem têm se tornado conceitos fundamentais na Administração, nas suas dis- 
ciplinas e em outras ciências, uma vez que, a partir de meados do século $\mathrm{XX}$ e início do século XXI, esses constructos têm competido em importância com o próprio capital financeiro.

Bennet e Tomblin (2006) afirmavam que, devido ao crescente reconhecimento da importância do conhecimento como recurso estratégico para as organizações modernas, não é de se estranhar que muitas áreas da Administração e de outras ciências se concentrem em sua criação, transformação, aprendizagem, uso e gestão.

Em relação à Aprendizagem Organizacional, cabe ressaltar que é dinâmica e multifacetada. São diversas abordagens do tema que envolvem dimensões distintas como psicológica, sociológica, cultural, histórica, metodológica, além da gestão propriamente dita (ANTONELO; GODOY, 2010).

Na mesma linha, Bido e Felix (2011) indicam que a AO tem sido pesquisada sob diversos enfoques: psicológico, sociológico, das ciências da Administração etc. Segundo os autores, sua definição varia de acordo com o enfoque adotado, o que é observado, de modo mais concreto, nos referenciais teóricos escolhidos pelos pesquisadores. Eles afirmam ainda que, apesar de as abordagens diferirem entre si, pode-se identificar certa sobreposição de definições constitutivas e operacionais para o conceito.

Easterby-Smith (1997) traz seis perspectivas acadêmicas que entende terem potencial para explicar, em parte, a AO: psicologia, ciências da administração, estratégia, administração da produção, sociologia e antropologia cultural.

Neste artigo, tanto as perspectivas da administração, da produção e das ciências da administração foram consideradas perspectivas das ciências da administração, e as perspectivas política e cultural foram apresentadas em conjunto.

A perspectiva psicológica, para Bastos, Gondim e Loiola (2004), parte da premissa de que a aprendizagem ocupa um lugar de destaque nas teorias psicológicas e constitui um processo amplo e complexo, pois está intimamente relacionada a uma abordagem cognitivista, a fatores intra e interpsíquicos. Para os autores, essa é uma abordagem teórica que explora o tema a partir do entendimento de que aprender é uma mudança comportamental e de atitude que envolve os planos afetivo, motor e cognitivo. 
Ao dialogar sobre a faceta psicológica da AO, Lipshitz, Popper e Friedman (2002) afirmam que o aprendizado organizacional produtivo é bastante raro, porque requer dois estados psicológicos que são difíceis de manter. O primeiro estado é a segurança psicológica, sem a qual as pessoas relutam em assumir os riscos necessários à aprendizagem; o segundo é o comprometimento organizacional, sem o qual as pessoas ficam relutantes em compartilhar informações e conhecimentos com outros indivíduos.

Para Bastos, Gondim e Loiola (2004), a psicologia e suas disciplinas têm contribuído para as discussões que estão sendo desenvolvidas em Aprendizagem Organizacional. Contudo, um dos grandes desafios com que os interessados da área se deparam é a adequada transposição de conhecimentos produzidos na psicologia individual para o contexto organizacional.

Já a perspectiva da ciência da administração traz a importância da informação para o contexto da AO. Para um indivíduo ou grupo tomarem uma decisão de maneira eficiente, devem ter condições de armazenar e tratar essas informações. Huber e March (1991) propõem quatro construtos relacionados com AO: aquisição de conhecimento, distribuição de informação, interpretação da informação e memória organizacional.

Vê-se uma aproximação dos conceitos da $\mathrm{AO}$ com os da gestão do conhecimento, uma vez que a relação entre aprendizagem e conhecimento é tratada nesta perspectiva: primeiro o conhecimento deve ser adquirido para depois ser repassado para toda a organização. Essa distribuição pode levar ao desenvolvimento de novos conhecimentos, perspectiva que aproxima a AO das ciências da informação, uma vez que traz a informação e seus sistemas para o cerne das discussões.

A aprendizagem, segundo o enfoque da perspectiva sociológica e teoria organizacional, é construída nas relações entre pessoas e grupos. Segundo Antonelo e Godoy (2010, p. 314), “[...] nesta dimensão os aspectos situacionais da aprendizagem têm papel central, enquanto os princípios psicológicos da cognição social e individual são considerados processos secundários".

Pode-se então compreender aprendizagem como processo social. Dessa forma, os indivíduos ou grupos nunca aprendem sozinhos; 
na verdade, nunca estão sozinhos, mas em constante relação dialógica. Estudar aprendizagem a partir dessa perspectiva é analisar as relações.

Easterby-Smith (1997) propõe que essa abordagem pode ser dividida em: funcionalista, de contingência e construtivista.

A abordagem funcionalista parte da premissa de que os aspectos estruturais, especialmente onde mais se aproximam do modelo burocrático, são fatores que podem dificultar ou facilitar a aprendizagem. Além da estrutura, dimensões políticas, culturais e hierárquicas influem no processo de AO.

A visão da contingência, segundo Easterby-Smith (1997), sugere que a $\mathrm{AO}$ significa coisas diferentes e opera de maneiras díspares, de acordo com a natureza da organização. A aprendizagem é reconhecida como um processo que se passa dentro da organização e torna-se propriedade de todos os seus membros.

A abordagem construtivista e critica sugere que a aprendizagem informal está em oposição à formal. Na análise de Nicolini e Meznar (1995), o construtivismo tem uma perspectiva mais ampla e o conhecimento é construído e reconstruído seguindo descontinuidades percebidas no funcionamento organizacional.

A perspectiva antropológica cultural aponta para a cultura como algo importante no processo de AO. A cultura organizacional é fator determinante em vários processos e as relações serão estabelecidas por meio da cultura.

Sobre a faceta cultural da AO, Friedman et al. (2005) identifica cinco normas que são suscetíveis de produzir informações válidas, além de um compromisso com a ação corretiva: transparência, integridade, issue orientation, inquiry e accountability. Trata-se de manifestações observadas em um conjunto de valores comuns que constituem uma cultura organizacional propícia à aprendizagem produtiva.

Para Friedman et al. (2005), a transparência é definida como expor seus pensamentos e ações para os outros, a fim de receber feedback. A integridade é definida pela disponibilização de informação, independentemente de suas implicações. Issue orientation é definida como a relevância da informação para as questões, independentemente da posição social ou da posição do destinatário ou da fonte. Inquiry, questionamento, é definido como a persistência na investi- 
gação até que a plena compreensão seja alcançada. Accountability é definida como assumir a responsabilidade pelo que foi aprendido.

A abordagem cultural trata de uma forma de pensar em que a AO tem forte ligação com a perspectiva sociológica, uma vez que a cultura representa uma sociedade e os grupos sociais.

Por fim, a aprendizagem sob o ponto de vista da perspectiva estratégica pode ser pensada a partir de sua relação com a aquisição de vantagens competitivas, ou seja, a $\mathrm{AO}$ é um dos fatores que alinha a organização e o ambiente interno e externo. A aprendizagem pode, então, ser pensada como uma dentre as formas que a organização possui de se diferenciar de sua concorrência de maneira estratégica.

Para Levinthal e March (1993), o aprendizado tem sido “descoberto" pelo mundo da prática e do campo acadêmico da gestão estratégica. Como os pesquisadores têm considerado a estabilidade das diferenças de desempenho da empresa em face da mudança de ambientes de negócios, muitos passaram a ver a capacidade de aprender como um importante fator; em alguns casos, a única fonte sustentável de vantagem competitiva.

\subsection{Redes semânticas}

Sowa (1991) considera redes semânticas como estruturas de representação do conhecimento formadas por vértices e arestas. Para o autor, os primeiros registros de redes semânticas remontam à década de 1960 e estavam relacionados principalmente às Ciências da Computação.

Pode-se pensar em uma rede semântica como aquela formada por frases, palavras, bigramas e n-gramas que têm conteúdo semântico, "significado". As relações entre essas frases ou palavras podem ser analisadas quanto à sua estrutura.

A Análise de Redes Semânticas (ARSe) surgiu nas duas últimas décadas com a preocupação de estudar grandes volumes de textos. Essa forma de interpretação textual consiste em representar o conteúdo de uma mensagem na forma de uma rede de objetos. Várias têm sido as pesquisas em redes semânticas. A seguir, serão apresentados alguns autores que se utilizam delas.

Para Lee et al. (2013), a rede semântica é um gráfico de conceitos. Já a Análise de Redes Semânticas (ARSe) é uma filial das Análises de Redes Sociais (ARS) que exploram as relações entre significados, compartilhados em configurações linguísticas sociais. A ARSe pode, a exemplo da ARS, ser feita por várias métricas como: 
a densidade da rede, a centralidade da rede, o grau de centralidade, betweenness centralidade, eigenvector centralidade, comprimento de camino e outras.

A seguir, serão discutidas e apresentadas algumas estratégias metodológicas para pesquisar redes semânticas de artigos científicos.

\section{Metodologia}

Destaca-se que o método é quantitativo, bibliométrico, exploratório e longitudinal. A opção pela análise bibliométrica deveu-se ao fato de a pesquisa utilizar dados bibliográficos secundários no intuito de estudar as relações entre esses dados. Hackett e Borgman (1992), ponderando a respeito dos métodos bibliométricos, afirmam que podem ser utilizados no estudo da comunicação científica, assinalam que são métodos pelos quais as atividades de comunicação podem ser explicadas e interpretadas, considerando os objetos, agentes, eventos, produtos, e contextos de tal atividade como entidades a serem contadas, medidas ou quantificadas.

Para Hair Jr. et al. (2005), a pesquisa exploratória não tem a intenção de testar hipóteses ou preposições antes estabelecidas. Ela parte de um princípio em que o pesquisador busca, por meio da observação científica, explorar a área pesquisada. Segundo os autores, essa é uma técnica útil quando o pesquisador não sabe muito, ou seja, está orientado para a descoberta, não tem a intenção de testar hipóteses ou modelos preestabelecidos.

O estudo é longitudinal, pois busca, através do tempo, perceber como as redes semânticas têm se comportado. Hair Jr. et al. (2005) apontam que investigações longitudinais são importantes para observar tendências temporais, pois a dinâmica da pós-modernidade, aliada à produção e à circulação de grande volume de produção científica, deixa as análises atemporais estáticas e obsoletas.

O período de tempo investigado vai do ano de 1965 a 2015, pois o documento mais antigo encontrado na base de dados é de 1965.

\subsection{Estratégias de pesquisa em bases de dados}

Como exposto na introdução, a produção de documentos científicos na Administração e em outras ciências tem crescido muito nas últimas décadas. Além disso, o desenvolvimento e a posterior popularização das bases de dados de pesquisa contribuíram para a expansão do acesso a eles. O problema é que, devido a dificulda- 
des de indexação relativas ao aumento da oferta de documentos, localizar conjuntos de artigos que sejam relevantes tem se tornado uma tarefa cada vez mais difícil.

No intuito de solucionar ou ao menos amenizar o problema, propôs-se o desenvolvimento de uma estratégia de busca adaptada de Neder, Marques e Carvalho (2016) que seja funcional, mensurável e escalável para aplicá-la à área de $\mathrm{AO}$ como forma de garantir a confiabilidade e a abrangência dos dados analisados neste artigo.

Estratégia de busca pode ser definida, segundo Lopes (2002), como um conjunto de regras e técnicas que tornam possível o encontro da informação armazenada em uma base de dados. O autor aponta que para alcançar a resposta pretendida pelo pesquisador, faz-se necessária a execução de operações lógicas, ora restringindo os resultados alcançados, ora ampliando-os para obtenção de informação relevante para a pesquisa.

A pesquisa contemporânea em bases de dados se deve, em parte, ao trabalho de Eugene Garfield, que, segundo Wormell (1998), propôs a criação dos índices de citação. Tinha como objetivo melhorar a forma como acontecia a recuperação da informação científica. Continuando com a autora, havia, na época, uma insatisfação com os serviços de indexação: "Todos eram publicados com excesso de atraso, a indexação era inconsistente e descoordenada e as políticas de seleção deixavam grandes falhas na sua cobertura" (WORMELL, 1998, p. 210).

Considerando essas informações, foram efetuados os passos que estão representados na Figura 1. Primeiro foi necessário estabelecer um glossário dos termos que tinham potencial para recuperar o máximo de artigos da área de $\mathrm{AO}$; para tanto, foram consultados artigos de rewiew, handbooks da área, além, da base de dados de teste criada a partir da consulta do termo organizacional learning na base de dados do Web of Science. Esses passos permitiram estabelecer 24 termos iniciais que tinham relação com Aprendizagem Organizacional.

De posse desses termos foi possível testar a precisão de cada termo lendo o título e o resumos de $5 \%$ dos artigos recuperados na consulta de cada termo individualmente. Se o valor da precisão foi maior que $70 \%$, o termo foi adicionado à consulta.

Além da precisão, os termos foram testados individualmente com especialistas - nesse caso os especialistas são os autores dos artigos da base de dados de teste. Foi enviado um e-mail para cerca 
de 7.000 pesquisadores das áreas de Aprendizagem Organizacional, Organizações que Apreendem e Gestão do Conhecimento com cada termo para que o relacionassem com a área do conhecimento. Foram obtidas 30 respostas, e se a porcentagem de especialistas que consideraram o termo como da área de $\mathrm{AO}$ foi maior ou igual a 50\% o termo foi agregado à estratégia.

Por fim, foi testada a revocação que indica a abrangência da consulta, ou seja, os artigos recuperados pela estratégia equivalem a pelo menos $70 \%$ dos artigos de $\mathrm{AO}$ presentes na base de dados consultada; em caso positivo, a consulta foi validada.

Figura 1: Estratégia para desenvolvimento da lista de termos para as buscas

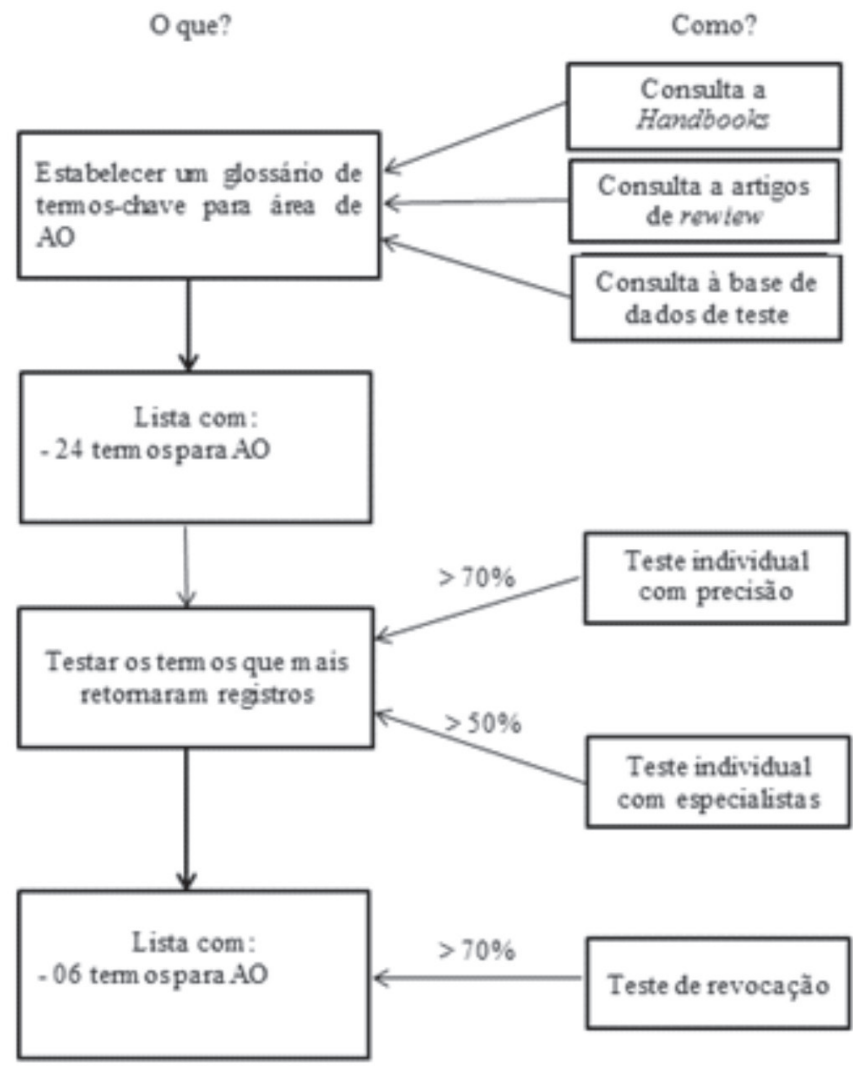

Fonte: Elaborado pelos autores. 
Innocentini, Bessi e Milanez (2014) apontam que a precisão pode ser medida pela Equação 1:

Equação 1

$$
\text { Precisão }=\frac{N^{\circ} \text { de referências relevantes recuperadas }}{N^{\circ} \text { de referêcias recuperadas pelo sistema }}
$$

Fonte: Innocentini, Bessi e Milanez (2014).

Innocentini, Bessi e Milanez (2014) trazem que a revocação pode ser medida pela Equação 2:

Equação 2

$$
\text { Revocação }=\frac{\mathrm{N}^{\circ} \text { de referências relevantes recuperadas }}{\mathrm{N}^{\circ} \text { de referêcias relevantes existentes no sistema }}
$$

Fonte: Innocentini, Bessi e Milanez (2014).

O Quadro 1 traz os seis termos usados na consulta final.

\begin{tabular}{|c|c|c|c|c|c|}
\hline \multicolumn{6}{|c|}{ APRENDIZAGEM ORGANIZACIONAL } \\
\hline \multicolumn{2}{|c|}{ "Organizational Learning"(\%) } & \multicolumn{2}{|c|}{ "Organisational Learning" (\%) } & \multicolumn{2}{|c|}{ "Collective Learning" (\%) } \\
\hline $\begin{array}{l}\text { E specialistas: } \\
\text { AO: } 93.9 \\
\text { OA: } 36.4 \\
\text { GC: } 15.2\end{array}$ & $\begin{array}{l}\text { Dados tese: } \\
\text { AO: } 64 \\
\text { Resultado: } \\
\text { Incluir }\end{array}$ & $\begin{array}{l}\text { E specia listas: } \\
\text { AO: } 93.9 \\
\text { OA: } 27.3 \\
\text { GC: } 18.2\end{array}$ & $\begin{array}{l}\text { Dados tese: } \\
\text { AO: } 81.97 \\
\text { Result ado: } \\
\text { Incluir }\end{array}$ & $\begin{array}{l}\text { E specialista s: } \\
\text { AO }: 66.7 \\
\text { OA: } 63.6 \\
\text { GC: } 18.2\end{array}$ & $\begin{array}{c}\text { Dados tese: } \\
\text { AO:70 } \\
\text { Resultad o: } \\
\text { Incluir }\end{array}$ \\
\hline \multicolumn{2}{|c|}{ "Loop Learning" (\%) } & \multicolumn{2}{|c|}{ "Operational Learning" (\%) } & \multicolumn{2}{|c|}{ "Team Learning" (\%) } \\
\hline $\begin{array}{l}\text { E specialista s: } \\
\mathrm{AO}: 72.7 \\
\mathrm{OA}: 39.4 \\
\mathrm{GC}: 12.1\end{array}$ & $\begin{array}{l}\text { Dados tese: } \\
\text { AO: } 80 \\
\text { Resultado: } \\
\text { Incluir }\end{array}$ & $\begin{array}{l}\text { Especia listas: } \\
\text { AO: } 57.6 \\
\text { OA: } 34.4 \\
\text { GC: } 12.1\end{array}$ & $\begin{array}{l}\text { Dados tese: } \\
\text { AO: } 80 \\
\text { Resultado: } \\
\text { Incluir }\end{array}$ & $\begin{array}{l}\text { E specialista s: } \\
\text { AO: } 90.9 \\
\text { OA: } 60.6 \\
\text { GC: } 36.4\end{array}$ & $\begin{array}{c}\text { Dados tese: } \\
\text { AO:90 } \\
\text { Resultad o: } \\
\text { Incluir }\end{array}$ \\
\hline
\end{tabular}

Quadro 1: Resultados da estratégia de busca

Fonte: Elaborado pelos autores.

Nota: Os termos foram encontrados principalmente nas seguintes fontes: Aprendizagem Organizacional: Argote, Miron-Spektor (2011), Conlon (2004), Crossan, Maurer e White (2011), Easterby-Smith (2000), Easterby-Smith e Lyles (2011), Hotho, Lyles e Easterby-Smith (2015) e Levitt e March (1988). Além das fontes elencadas, foram feitas consultas nos registros do banco de dados de teste com o intuito de verificar as palavras-chave dos artigos das três áreas. O download dos artigos foi realizado em dezembro de 2015. 
A limpeza dos dados foi executada por meio do software Automap (http://www.casos.cs.cmu.edu) que permitiu excluir a pontuação, caracteres especiais, pronomes, letras avulsas, preposições, números, datas e trazer os verbos para o infinitivo; além disso, o software permitiu ainda gerar listas de deleção (delete list) e listas de bigramas. As análises foram feitas utilizando o software ORA (http://netanomics.com/).

\subsection{Métricas}

Para Chen, Sanjuan e Hou (2010), a métrica centralidade pode ser definida para cada nó da rede e é medida a partir do grau em que o nó está no meio do percurso que o liga aos outros vértices dessa rede.

Newman (2005) assinala que betweenness é uma medida da centralidade de um nó em uma rede normalmente calculada como a fração de caminhos mais curtos entre pares de nós que passam pelo nó de interesse. É, em certo sentido, uma medida da influência que um nó tem sobre a disseminação da informação ou de outros conteúdos através da rede. Valores altos para essa métrica, segundo Chen, Sanjuan e Hou (2010), podem identificar publicações científicas potencialmente revolucionárias, bem como gatekeepers em redes sociais. Por exemplo, se um nó proporciona a única conexão entre dois grupos grandes, mas de outra forma não relacionados, ele teria um valor muito elevado de centralidade.

Pode-se definir centralidade como a propriedade de um nó ou de um grupo de nós que dizem respeito à sua posição em uma rede (BORGATTI; EVERETT; JOHNSON, 2013). Para os autores, pensar centralidade significa tentar compreender a contribuição que um nó ou conjunto de nós fazem à estrutura dessa rede, ou seja, a centralidade é o grau de importância estrutural de um nó em relação à rede.

Nesta pesquisa, a métrica de centralidade utilizada foi a $\mathrm{Be}$ tweenness Centrality, pois segundo Chen, Sanjuan e Hou (2010), ela tem potencial para indicar vértices importantes para as redes e esse grau de importância, medido ao longo do tempo, permite a estimativa de suas tendências. 


\section{Resultados}

$\mathrm{Na}$ análise dos dados, foram utilizados 2.896 artigos de AO. Percebe-se, por meio da visualização da rede demonstrada na Figura 2, que, apesar de ser uma fração dos textos de Aprendizagem Organizacional, ela traz uma dificuldade para interpretação devido à complexidade de suas relações; por esta razão utilizam-se métricas para sua análise.

Figura 2: Rede semântica de artigos de $A O$

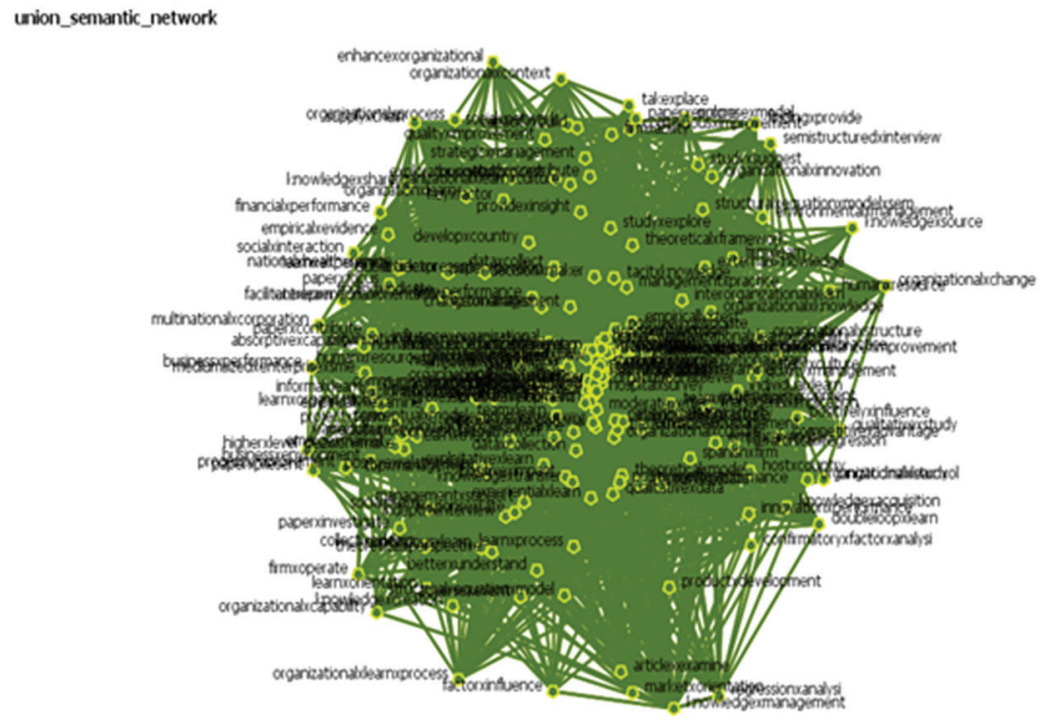

Nota: Redes de artigos de Aprendizagem Organizacional nos anos 2011- 2015.

Fonte: Resultado da pesquisa.

Foram geradas redes ano a ano, redes de períodos de tempo que continham o somatório dos artigos que pertenciam aos anos do período estudado e redes acumuladas que continham o somatório dos artigos dos anos anteriores.

Como exposto na introdução deste artigo, encontrar tendências que permitam demonstrar ou ao menos estimar o desenvolvimento de determinada área ou ciência pode permitir aos pesquisadores, 
grupos, agências de fomento, governos etc. tomarem decisões, com base em informação referenciada, sobre a melhor maneira de se aplicarem os recursos de pesquisa, além de permitir melhor compreensão de uma área de conhecimento.

Para fins de comparação dos textos das áreas, foram escolhidos os bigramas/trigramas com maior Betweenness Centrality (por área). Para tanto, foi criada uma rede semântica que continha exclusivamente os textos dos últimos dois anos da área de $\mathrm{AO}$. Os bigramas/trigramas encontrados ali foram então comparados com os das redes dos anos anteriores. Foram geradas redes semânticas de duas formas: na primeira (ano a ano), para cada ano, era criada uma rede independente dos outros anos; na segunda (acumulada), cada rede continha uma acumulação dos anos anteriores. Foi gerada uma curva de tendência que permite estimar o progresso de cada termo analisado nos anos subsequentes.

A seguir, o Quadro 2 apresenta os resultados das tendências da AO. Quando as duas tendências são positivas, pode-se aferir que a tendência geral de ampliação da métrica Betweenness Centrality ao longo do tempo também é positiva. Da mesma forma, quando são negativas, afere-se que a tendência geral é negativa. Os outros casos são de tendências neutras, quando as duas tendências permanecem estáveis ou não são passíveis de serem estimadas (uma tendência positiva e outra negativa).

As Figuras 3 e 4 apresentam, respectivamente, as curvas de tendência dos termos Innovation Performance e Organizational Learn Culture. Eles foram destacados, pois, apesar do seu relativo pouco tempo figurando nas redes semânticas de AO, mostram-se importantes para elas, o que pode ser aferido pela métrica Betweenness Centrality. Esse conjunto de fatores pode ser importante para identificar o surgimento de tópicos emergentes de pesquisa.

A seguir, são apresentados os gráficos de evolução da métrica Betweenness Centrality ao longo do tempo para os termos destacados. 
Quadro 2: Tendências para os termos de Aprendizagem Organizacional

\begin{tabular}{|c|c|c|c|}
\hline $\begin{array}{l}\text { TERMOSLEARNING } \\
\text { ORGANIZATION }\end{array}$ & OCORRÊNCLA & $\begin{array}{l}\text { TENDÊNCIA } \\
\text { ACUMULADA }\end{array}$ & $\begin{array}{l}\text { TENDÊNCLA } \\
\text { ANOA ANO }\end{array}$ \\
\hline \multicolumn{4}{|c|}{ Tópicos emergentes } \\
\hline $\begin{array}{c}\text { Innovation Performance } \\
\text { Organizational Lecon Culture }\end{array}$ & $\begin{array}{l}5 \text { anos ou menos } \\
5 \text { anos ou menos }\end{array}$ & $\begin{array}{l}\text { Ascendente } \\
\text { Ascendente }\end{array}$ & $\begin{array}{l}\text { Ascendente } \\
\text { Ascendente }\end{array}$ \\
\hline \multicolumn{4}{|c|}{ Tendências positiv as } \\
\hline Organizational Learn Theory & 6 anos oumais & Ascendente & Ascendente \\
\hline Absorptive Capacity & 6 anos oumais & Ascendente & Ascendente \\
\hline $\begin{array}{c}\text { Supply Chain } \\
\text { Knowledge Share }\end{array}$ & $\begin{array}{l}6 \text { anos oum mais } \\
6 \text { anos oum ais }\end{array}$ & $\begin{array}{l}\text { Ascendente } \\
\text { Ascendente }\end{array}$ & $\begin{array}{l}\text { Ascendente } \\
\text { Ascendente }\end{array}$ \\
\hline Firm Performance & 6 anos oumais & Ascendente & Ascendente \\
\hline Team Learn & 6 anos oum ais & Ascendente & Ascendente \\
\hline Organizational Performance & 6 anos oumais & Ascendente & Ascendente \\
\hline Knowledge Trconsfer & 6 anos oumais & Ascendente & Ascendente \\
\hline Organizational Inrovation & 6 anos ou mais & Ascendente & Ascendente \\
\hline Social Capital & 6 anos oum ais & Ascendente & Ascendente \\
\hline Ongconizational Culture & 6 anos oumais & Ascendente & Ascendente \\
\hline Organizational Learn Literature & 6 anos oum ais & Ascendente & Ascendente \\
\hline Orgconizational Level & 6 anos oumais & Ascendente & Ascendente \\
\hline \multicolumn{4}{|c|}{ Tend ências descend entes } \\
\hline Learn Orgcnization & 6 anos oumais & Descendente & Descendente \\
\hline \multicolumn{4}{|c|}{ Tendências indefinidas ou estáv eis } \\
\hline Prochuct Development & 6 anos oumais & Estável & Estável \\
\hline Knowledge Mconagement & 6 anos oum ais & Estável & Estável \\
\hline $\begin{array}{c}\text { Leam Process } \\
\text { Competitive Achantage }\end{array}$ & $\begin{array}{l}6 \text { anos oum mais } \\
6 \text { anos ou mais }\end{array}$ & $\begin{array}{c}\text { Estável } \\
\text { Ascendente }\end{array}$ & $\begin{array}{l}\text { Descendente } \\
\text { Descendente }\end{array}$ \\
\hline Organizational Learn Process & 6 anos ou mais & Ascendente & Descendente \\
\hline $\begin{array}{c}\text { Knowledge Creation } \\
\text { Pefformance Improvement }\end{array}$ & $\begin{array}{l}6 \text { anos oum mais } \\
6 \text { anos oum mais }\end{array}$ & $\begin{array}{c}\text { Estável } \\
\text { Ascendente }\end{array}$ & $\begin{array}{l}\text { Estável } \\
\text { Estável }\end{array}$ \\
\hline Market Orientation & 6 anos oumais & Ascendente & Estável \\
\hline Learn Theory & 6 anos oumais & Estável & Ascendente \\
\hline
\end{tabular}

Fonte: Elaborado pelos autores.

Nota 1: Na etapa de limpeza os verbos são trazidos todos para o infinitivo e são formados os bigramas (termos com duas palavras) por meio do algoritmo de Steaming, por isso, neste quadro há termos como: Organizational Learn.

Nota 2: A coluna Tendência Acumulada apresenta a tendência para a métrica Betweenness Centrality, para redes formadas pelo acúmulo dos anos anteriores; já a coluna de Tendência Ano a Ano demonstra a tendência por meio de redes independentes por ano. 
Figura 3: Evolução da centralidade para o termo Innovation Performance nas redes de Aprendizagem Organizacional, a partir do ano de 2010

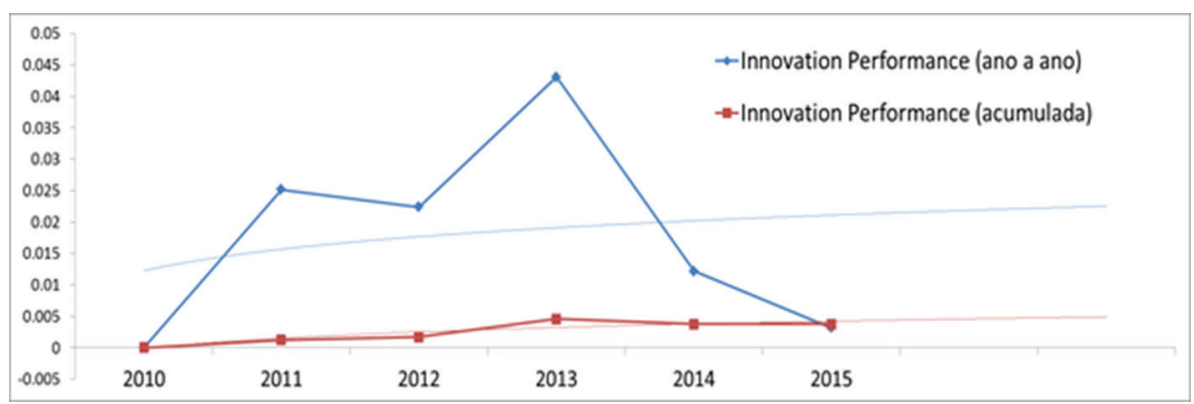

Fonte: Elaborado pelos autores.

O tópico de pesquisa foi considerado emergente quando figurava há cinco anos ou menos entre os 25 termos com maior Betweenness Centrality nas redes semânticas da área. A linha azul diz respeito à rede ano a ano e a linha vermelha às redes acumuladas.

Percebe-se no caso em questão uma tendência emergente até o ano de 2013, porém a tendência é decrescente a partir de 2014 para as redes ano a ano. Para a rede acumulada a tendência é estável a partir de 2014.

Figura 4- Evolução da centralidade para o termo Organizacional Learn Culture nas redes de Aprendizagem Organizacional, a partir do ano de 2010.

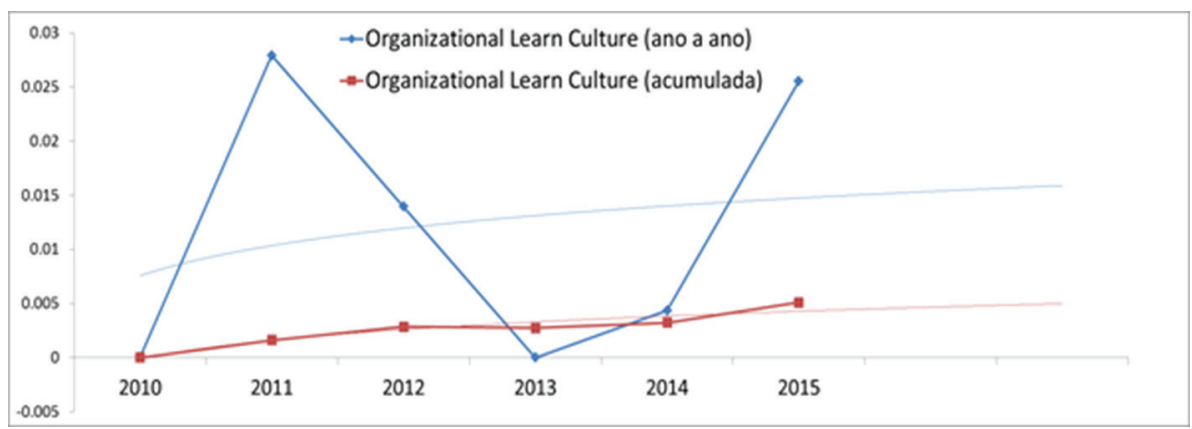

Fonte: Elaborado pelos autores.

Nota: A linha vermelha evidencia a Betweenness Centrality do termo na rede semântica dos anos acumulados (do ano respectivo ao mais antigo). A linha azul evidencia a Betweenness Centrality do termo em questão para redes ano a ano. 
Como fica evidente pela Figura 4, o termo Organizational Learn Culture demonstra uma tendência positiva em relação à métrica Betweenness Centrality.

\subsection{Crítica ao Modelo Easterby-Smith para AO}

A abordagem de Easterby-Smith (1997) traz seis perspectivas acadêmicas que, segundo o autor, tinham potencial, na época, para explicar a AO: psicologia, ciências da administração, estratégia, administração da produção, sociologia e antropologia cultural. Na contemporaneidade esse modelo não parece mais suficiente para categorizar a área. Por exemplo, quando se analisam os 18 termos mais importantes da rede de AO segundo a Betweenness Centrality nos anos de 2014-2015 (Quadro 3), poucas são as relações que se consegue fazer.

Quadro 3: Termos de Aprendizagem Organizacional com maior Betweenness Centrality para a rede de AO, nos anos de 2014-2015

\begin{tabular}{|c|c|c|}
\hline ITEM & BETWEENNESS CENTRALITY & $\begin{array}{c}\text { RELAÇÃO COM AS } \\
\text { ABORDAGENS }\end{array}$ \\
\hline knowledgex managem ent & 0.044350687 & $?$ \\
\hline leamxprocess & 0.035226688 & sociologia \\
\hline organizationalx learnx theory & 0.03473871 & $?$ \\
\hline absorptivex capacity & 0.033501275 & $?$ \\
\hline supplyx chain & 0.026429176 & admini stração da produção \\
\hline knowledgex share & 0.025487661 & $?$ \\
\hline firmxperformance & 0.024386678 & estratégia \\
\hline teamxlearn & 0.023942728 & sociologia \\
\hline organiz ationalx perform ance & 0.022119654 & sociologia \\
\hline productx development & 0.019348808 & admini stração da produção \\
\hline knowledgextransfer & 0.019182064 & ? \\
\hline learnx organization & 0.018155478 & $?$ \\
\hline organizationalx innovation & 0.017491924 & ? \\
\hline socialxcapital & 0.01617189 & ? \\
\hline innovationxperform ance & 0.015462698 & $?$ \\
\hline organizational xculture & 0.015430016 & antr op ologia cul tural \\
\hline competitivex advantage & 0.013795803 & estratégia \\
\hline organizationalxlearnxculture & 0.013206095 & antr opologia cultural \\
\hline
\end{tabular}

Fonte: Elaborado pelos autores.

Nota 1: Na etapa de limpeza os verbos são formados os bigramas (termos com duas palavras) por meio do algoritmo de Steaming, por isso, neste quadro há termos como: organizationalxculture.

Nota 2: 18 termos com maior centralidade na rede dos últimos dois anos de Aprendizagem Organizacional. Os bigramas e trigramas são apresentados juntos pois o software de análise tem que compreende-los enquanto uma unidade de sentido. Um n-grama é um conjunto de palavras que forma uma unidade de sentido. 
Pode-se identificar, pelo Quadro 3, que o modelo proposto por Easterby-Smith em 1997 é incompleto para categorizar a produção dos últimos dois anos de $\mathrm{AO}$ presente na base de dados Web of Science. Para atualizá-lo, seria necessário, por exemplo, incluir perspectivas que abarquem textos de $\mathrm{AO}$ que têm relação com Inovação e Gestão do Conhecimento.

\section{CONSIDERAÇõES FINAIS}

Uma rede semântica formada por mais de 2.896 artigos científicos, em que suas relações de conteúdo apresentam muitos milhares de arestas e alguns milhares de vértices, pode parecer, à primeira vista, um emaranhado confuso e não inteligível de relações. Porém, as análises de redes sociais e semânticas funcionam como uma bússola e permitem descobrir tendências e caminhos, traçar rotas e corrigir percursos.

As pesquisas tradicionais que consideram dezenas ou, no máximo, poucas centenas de documentos, e não são viáveis para se lidar com um universo de milhares de artigos, uma vez que a análise de poucos textos pode não apresentar nuances importantes para a compreensão da área.

Portanto, a utilização de metodologias escaláveis, baseadas em tecnologias da informação que permitam analisar grandes quantidades de dados, é importante para tentar compreender a complexidade das ciências e de seus campos de estudo.

Dessa forma, foi possível atingir os objetivos propostos, que eram explorar a rede de Organizações que Aprendem, apontando os tópicos emergentes e tendências.

Entre as contribuições teóricas deste artigo cabe apontar as frentes de pesquisa que têm potencial para se destacar na área. Foram encontradas diversas delas que estão em uma curva de ascendente, como pôde ser visto no Quadro 2. Além disso, foram apresentados os termos Innovation Performance e Organizational Learn Culture para as redes de $\mathrm{AO}$ como possíveis tópicos emergentes para a área de AO. Foi possível ainda fazer uma crítica ao modelo de Easterby -Smith (1997) que se demonstra ultrapassado para categorizar os termos encontrados nestas análises. 
A respeito das contribuições metodológicas, as estratégias empregadas neste artigo se mostraram úteis e importantes para se trabalhar com pesquisa social em administração e em outras ciências, sobretudo quando o universo de dados for extenso e complexo. O artigo desmistifica as técnicas de Análise de Redes Semânticas, ainda pouco usadas no Brasil e no mundo, podendo, portanto, servir como um framework metodológico que auxilie pesquisadores das mais diversas áreas a trilhar alguns dos caminhos aqui percorridos.

$\mathrm{O}$ recorte temporal permitiu estimar tendências, apontar possíveis frentes emergentes de pesquisa e assim traçar caminhos plausíveis que $\mathrm{AO}$ pode vir a percorrer.

As contribuições práticas apontam para a utilização das técnicas, metodologias e resultados obtidos como forma de balizar pesquisadores, instituições, grupos de pesquisa e agências de fomento na alocação de seus recursos de pesquisa.

Propõe-se como pesquisas futuras examinar a mesma metodologia em textos de Gestão do Conhecimento e ainda analisar as relações entre Gestão do Conhecimento, Aprendizagem Organizacional e Organizações que Aprendem por meio das análises de redes semânticas.

\section{REFERÊNCIAS}

ANTONELO, C. S.; GODOY, A. S. A encruzilhada da Aprendizagem Organizacional: uma visão multiparadigmática. Revista de Administração Contemporânea, Curitiba, v. 14, n. 2, p. 310-332, 2010.

ARGOTE, L.; MIRON-SPEKTOR, E. Organizational learning: From experience to knowledge. Organization Science, [s.1.], v. 22, n. 5, p. 1123-1137, 2011.

BASTOS, B.; GONDIM, S. M.; LOIOLA, E. Aprendizagem organizacional versus organizações que aprendem: características e desafios que cercam essas duas abordagens de pesquisa. Revista de Administração da Universidade de São Paulo, São Paulo, v. 39, n. 3, p. 220-230, 2004.

BENNET, A.; TOMBLIN, M. S. A learning network framework for modern organizations: organizational learning, knowledge management and ICT support. VINE Journal of Information and Knowledge Management Systems, [s.1.], v. 36, n. 3, p. 289-303, 2006.

BIDO, S.; FELIX, B. Comparação de três escalas para a mensuração da Aprendizagem Organizacional. In: ENCONTRO DA ANPAD (ENANPAD), 35., 2011, Rio de Janeiro. Anais... Rio de Janeiro: ANPAD, 2011. p. 1-17.

BORGATTI, P. S.; EVERETT, G. M.; JOHNSON, C. J. Analyzing social networks. London: SAGE Publications Limited, 2013. 
CONLON, T. J. A review of informal learning literature, theory and implications for practice in developing global professional competence. Journal of European industrial training, [s.l.], v. 28, n. 4, p. 283-295, 2004.

CHEN, C.; SANJUAN, F. I.; HOU, J. The structure and dynamics of cocitation clusters: a multiple-perspective cocitation analysis. Journal of the American Society for Information Science and Technology, [s.1.], v. 61, n. 7, p. 1386-1409, 2010.

CROSSAN, M. M.; MAURER, C. C.; WHITE, R. E. Reflections on the 2009 AMR decade award: do we have a theory of organizational learning? Academy of Management Review, [s.l.], v. 36, n. 3, p. 446-460, 2011.

EASTERBY-SMITH, M. Disciplines of organizational learning: contributions and critiques. Human Relations, [s.1.], v. 50, n. 9, p. 1085-1113, 1997.

. Organizational learning: debates past, present and future. Journal of Management Studies, [s.1.], v. 37, n. 6, p. 783-796, 2000.

EASTERBY-SMITH, M.; LYLES, M. A. Handbook of organizational learning \& knowledge management. 2. ed. Reino Unido: Wiley, 2011.

FRIEDMAN, V. J.; LIPSHITZ, R.; POPPER, M. The mystification of organizational learning. Journal of Management Inquiry, [s.1.], v. 14, n. 1, p. 19-30, 2005.

FUJITA, K.; KAJIKAWA, Y.; MORI, J.; SAKATA, I. Detecting research fronts using different types of weighted citation networks. Journal of Engineering and Technology Management, [s.l.], v. 32, p. 129-146, 2014.

GROH, G.; FUCHS, C. Multi-modal social networks for modeling scientific fields. Scientometrics, [s.1.], v. 89, n. 2, p. 569-590, 2011.

HACKETT, E. J.; BORGMAN, C. L. Scholarly communication and Bibliometrics. Contemporary Sociology, [s.1.], v. 21, n. 1, p. 142, 1992.

HAIR JR, J. et al. Fundamentos de métodos de pesquisa em Administração. Porto Alegre: Bookman, 2005.

HOTHO, J. J.; LYLES, M. A.; EASTERBY-SMITH, M. The mutual impact of global strategy and organizational learning: current themes and future directions. Global Strategy Journal, [s.l.], v. 5, n. 2, p. 85-112, 2015.

HUBER, G. P.; MARCH, J. G. Organizational learning: the contributing processes and the literatures. Organization Science, [s.1.], v. 2, n. 1, p. 88-115, 1991.

INNOCENTINI, L. L. F.; BESSI, N. C.; MILANEZ, D. H. Indicadores tecnológicos: estratégia de busca de documentos de patentes relacionados à instrumentação aplicada ao agronegócio. CC\&T - Cadernos de Ciência \& Tecnologia, [s.1.], v. 31, n. 1, p. 119-144, 2014.

LEE, H. et al. The moderating role of socio-semantic networks on online buzz diffusion. Journal of Business Research, [s.1.], v. 66, n. 9, p. 1367-1374, 2013.

LEVINTHAL, D. A.; MARCH, J. G. The myopia of learning. Strategic Management Journal, [s.l.], v. 14, n. 2, p. 95-112, 1993. 
LEVITT, B.; MARCH, J. G. Organizational learning. Annual Review of Sociology, [s.l.], v. 14, p. 319-340, 1988.

LIPSHITZ, R.; POPPER, M.; FRIEDMAN, V. J. A multifacet model of organizational learning. The Journal of Applied Behavioral Science, [s.1.], v. 38, n. 1, p. 78-98, 2002.

LOPES, I. L. Uso das linguagens controlada e natural em bases de dados: revisão da literatura. Ciência da Informação, [s.1.], v. 31, n. 1, p. 41-52, 2002.

NEDER, R.; MARQUES, J. C.; CARVALHO, A. S. C. Estratégia de Pesquisa em Bases de Dados. Espacios, Caracas, v. 37, n. 7, p. 1-15, 2016.

NICOLINI, D.; MEZNAR, M. B. The social construction of organizational learning: conceptual and practical issues in the field. Human Relations, [s.1.], v. 48, n. 7, p. 727-746, 1995.

SHIBATA, N.; KAJIKAWA, Y. Measuring relatedness between communities. Journal of the American Society for Information Science and Technology, [s.1.], v. 62, n. 7, p. 1360-1369, 2011.

SOWA, J. F. Principles of semantic networks. California: Morgan Kaufmann Publishers, 1991.

TAKAHASHI, A. R. W. Competências, Aprendizagem Organizacional e Gestão do Conhecimento. Curitiba: Intersaberes, 2015.

WORMELL, I. Informetria: explorando bases de dados como instrumentos de análise. Ciência da Informação, [s.1.], v. 27, n. 2, p. 210-216, 1998.

Recebido em: 07-01-2017

Aprovado em: 24-04-2017

Avaliado pelo sistema double blind review.

Editor: Elmo Tambosi Filho

Disponível em http://mjs.metodista.br/index.php/roc 